\title{
La evaluación del desempeño docente en la educación superior
}

\author{
María Luisa del Carmen Pacheco Cámara, Isela Ibarra Bocardo, \\ Miriam Elizabeth Iñiguez Galindo, Héctor Lee García y \\ Claudia Victoria Sánchez
}

\section{Resumen}

La evaluación del desempeño docente es un ejercicio sistemático que, con fundamento en un conjunto de evidencias, juzga cómo llevan a cabo su labor los profesores en las aulas, los talleres, los laboratorios o espacios de práctica, con el fin de identificar logros y desajustes de su actuación en los escenarios educativos. La valoración del desempeño de los docentes puede sustentarse en diversos modelos que varían en sus marcos, conceptos y procedimientos establecidos, todos tienen sin duda la finalidad de aportar evidencias que faciliten y promuevan la mejora de la enseñanza para contribuir a una educación de calidad.

El presente artículo es de carácter introductorio, en él se exponen definiciones generales de la docencia y el desempeño docente, así como las razones y formas de evaluarlo. Se pretende brindar un panorama básico al lector, a partir de la experiencia y el conocimiento de los autores.

Palabras clave: docencia, desempeño docente, evaluación, modelos de evaluación del desempeño docente. 


\section{THE EVALUATION OF TEACHER'S PERFORMANCE IN HIGHER EDUCATION}

\section{Abstract}

The evaluation of teacher's performance is a systematic exercise that, based on an evidence system, judges how teachers carry out their work in classrooms, workshops, labs or practice spaces, in order to identify achievements and shortcomings in their educational settings. The assessment of teacher's performance can be based on different models that differ in their frames, concepts and procedures, all of them have the purpose of providing evidence that facilitates and promotes teaching improvement and contributes to quality education.

This article has an introductory nature, it presents general definitions of teaching and teaching development, and the reasons and ways to evaluate it. It intends to provide a basic perspective based on the experience and knowledge of the authors.

Keywords:teaching, teaching performance, evaluation, teacher performance evaluation models.

\section{María Luisa del Carmen Pacheco Cámara}

luisadgee@comunidad.unam.mx

Maestra en Psicología por la Facultad de Psicología de la UNAM. Tiene experiencia laboral en reclutamiento, selección y capacitación de personal. Actualmente se especializa en evaluación educativa como Secretaria Auxiliar de Procesos y Programas Académicos en la Coordinación de Desarrollo Educativo e Innovación Curricular (CODEIC) de la UNAM, donde asesora y brinda apoyo técnico en evaluación docente, de planes y programas, así como en estudios de experiencias académicas y de seguimiento de egresados.

\section{Isela Ibarra Bocardo}

isela dgee@hotmail.com

Licenciada en Psicología con doce años de experiencia en evaluación de la docencia, la tutoría y la enseñanza, programas académicos y planes de estudio. Ha participado en los seminarios y prácticas en la residencia de la maestría en Evaluación Educativa e impartido cursos, talleres y asesorías a personal de instituciones educativas de la UNAM y otras universidades del interior de la república. También ha apoyado los esfuerzos que realizan los programas de licenciatura para llevar a cabo la acreditación. Actualmente se desempeña como Secretaria Auxiliar de Evaluación de Docencia y Tutoría, CODEIC, UNAM.

\section{Miriam Elizabeth Iñiguez Galindo}

igmiriam.unam@gmail.com

Licenciada en Psicología por la Universidad de Guadalajara y maestra en Psicología con Residencia en Evaluación Educativa de la UNAM. Ha colaborado en la Secretaría de Investigación y Estudios Avanzados de la UAEM, en los comités interinstitucionales para la evaluación de la educación superior. Actualmente se desempeña como jefa de la Unidad Académica de Acreditación y Seguimiento de Egresados de la CODEIC, UNAM. 


\section{Héctor Lee García}

Licenciado en Economía y candidato al grado de maestría en Ciencias Económicas por la Escuela Superior de Economía del IPN. Cuenta con 26 años de antigüedad en la UNAM. Actualmente forma parte del equipo de trabajo de la CODEIC, UNAM, como líder de proyecto de Estadística y Análisis de Datos, tiene a su cargo el análisis de datos de diversos servicios de evaluación que se dan a las facultades y escuelas de la institución.

\section{Claudia Victoria Sánchez}

victoriaeveedunam@gmail.com

Licenciada en Psicología, profesora de inglés certificada y egresada de la Maestría en Psicología con Residencia en Evaluación Educativa de la UNAM. Actualmente colabora como especialista en la CODEIC, UNAM, donde realiza actividades de evaluación docente, de planes y programas de estudio y estudios de seguimiento de egresados.

\section{La docencia y el desempeño docente}

En las instituciones de educación superior, la docencia se entiende como un ejercicio profesional que está a cargo del cuerpo académico de las instituciones y que tiene la finalidad de educar a los profesionales que la sociedad requiere. Es, sin duda, un ejercicio que se enmarca en un contexto institucional, académico y administrativo en el que la actuación de los docentes está sujeta a un sinnúmero de criterios, reglas y condiciones.

Por su naturaleza especializada, la docencia universitaria exige el dominio y manejo de múltiples saberes y habilidades, indispensables para orientar y apoyar las experiencias de aprendizaje de los estudiantes.

El ejercicio docente tiene que sustentarse en una doble formación de saberes, los de naturaleza disciplinaria y los de índole estrictamente pedagógica. Los primeros representan la materia o materias objeto de enseñanza y abarcan un conjunto de conocimientos específicos de una o varias disciplinas; mientras que los segundos, los pedagógicos, se refieren al conocimiento y comprensión de lo educativo en sus dimensiones filosóficas, teóricas, metodológicas y técnicas, para lograr síntesis singulares que posibiliten a los docentes formar individuos en escenarios de aprendizaje controlados.

Existen otras condiciones que también enmarcan el ejercicio docente, como el nivel educativo —licenciatura, especialización, maestría, doctorado-; la modalidad en la que se entrega el servicio educativo - presencial, abierta, a 
Figura 1. Condiciones que enmarcan el ejercicio docente. Imagen: Victoria Sánchez. Diseño gráfico: Julián Cruz Cruz.

distancia o mixta-; las normas operativas de los programas educativos, entre otras más, que obligan a los docentes a dominar conocimientos diferenciales, asumir estilos de enseñanza variados y realizar numerosas tareas de supervisión y apoyo al momento de operar la docencia en el escenario educativo (ver figura 1).

\section{Condiciones que enmarcan el ejercicio docente}
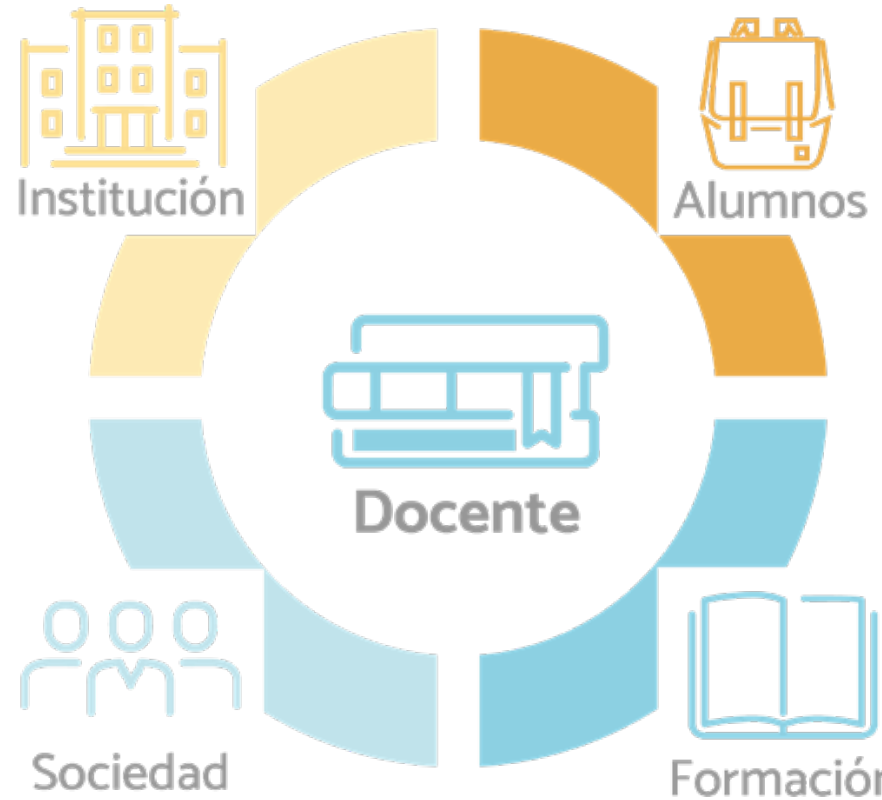

Docente

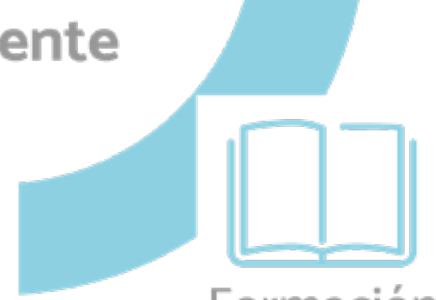

A lo largo del tiempo, a quienes ejercen la docencia se les han ido confiriendo otros roles, producto de los cambios del entorno social y de nuevas demandas que emergen; así como debido a las características y nuevos perfiles de las poblaciones de estudiantes; a la incorporación de la tecnología en diversos planos de los escenarios sociales, educativos y laborales; también a transformaciones de las mismas instituciones educativas, y sin duda, al desarrollo de nuevas pedagogías y recursos, entre otros muchos factores y presiones de cambio.

Ser un profesional de la docencia hoy en día es un reto extraordinario. Los docentes son, sin lugar a dudas, piezas clave del compromiso educativo que una institución asume con la sociedad; por ello, es necesario que se reconozca el valor de su contribución social; sus acciones orientadas a la producción y transformación del conocimiento; su trabajo en la promoción de valores entre los integrantes de las nuevas generaciones; su acompañamiento en el crecimiento, formación de la personalidad y desarrollo de la capacidad de sus alumnos para tomar decisiones; en suma, su aportación a la mejora de la sociedad, pues educar conlleva el valor y la fuerza de transformarla.

\section{La evaluación del desempeño docente}

La evaluación del desempeño docente debe ser un ejercicio sistemático que, basado en la recopilación de evidencias, ayude a las instituciones educativas a valorar tanto los aciertos como los logros del quehacer de estos actores, así 
como a identificar las áreas o aspectos en los que su desempeño es limitado o deficitario. Sin duda, la finalidad de este proceso es fortalecer las instituciones para cumplir un compromiso con la calidad de su esfuerzo educativo.

Toda evaluación del desempeño docente es compleja porque exige reconocer y comprender una amplia gama de factores que intervienen en la actuación del profesor. Cada proceso de evaluación, por otro lado, es único ya que obedece a un contexto, aunque, al mismo tiempo, tiene que atender la lógica de cualquier ejercicio profesional de evaluación en el que los demandantes, junto con los evaluadores expertos, deben precisar los propósitos de la evaluación del desempeño docente; definir el marco de referencia; decidir los enfoques, modelos e instrumentos que serán empleados; y orquestar toda la trama de tareas que permiten instrumentar una evaluación para integrar un sistema de evidencias.

Para realizar una evaluación del desempeño docente es indispensable delimitar el perfil del buen docente en un contexto institucional, es decir, deben definirse los conocimientos, habilidades y actitudes que idealmente tendría que dominar un profesor (ver figura 2). La experiencia acumulada a través de sucesivas investigaciones y ejercicios de evaluación ha conducido a identificar aspectos comunes que definen el perfil de un buen docente (Dewar, 2002; Coe et al., 2014), entre los que destacan el dominio de los conocimientos de las asignaturas que se imparten, los métodos y estrategias de enseñanza, el compromiso del docente en el escenario educativo y la relación que establece con los estudiantes. No obstante, no se ha alcanzado un acuerdo universal y, por lo tanto, en cada proceso de evaluación hay que definir un perfil de la docencia congruente con el ideario institucional, las condiciones de corte disciplinario y las necesidades de evaluación y uso que se dará a los resultados de este proceso.

\section{El perfil del desempeño docente}

Figura 2. El perfil del desempeño docente. Imagen: Victoria Sánchez. Diseño gráfico: Julián Cruz Cruz.

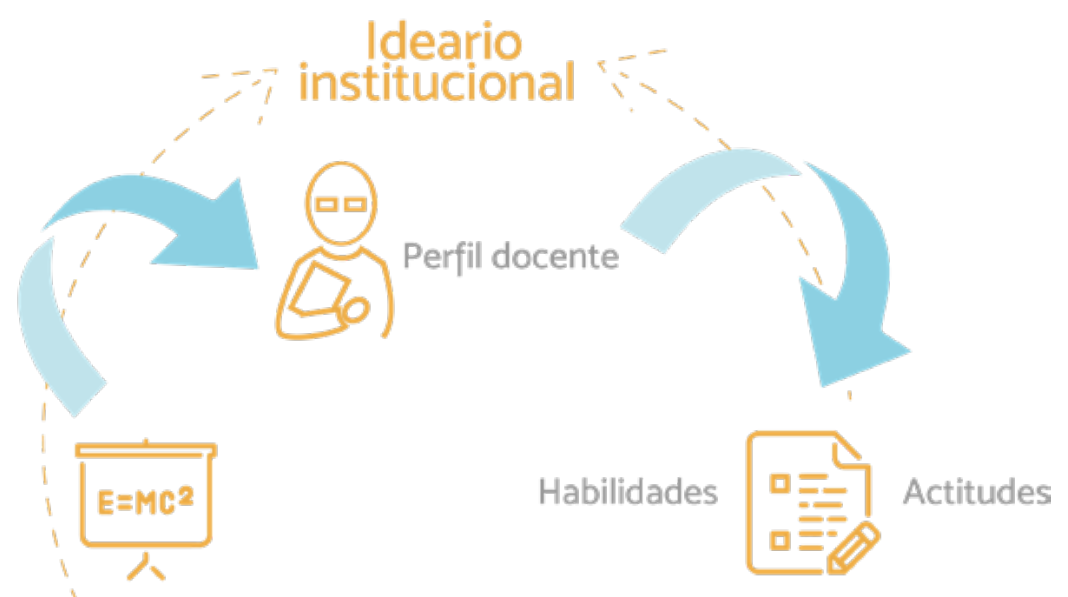

Dominios disciplinario Y pedagógico
Experiencia profesional

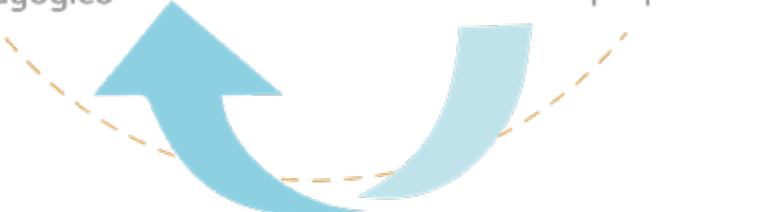


Por otro lado, es necesario definir con claridad el propósito de la evaluación; elegir uno o varios modelos de ésta; acordar un programa para el desarrollo del proceso; construir, probar y perfeccionar los instrumentos que serán empleados; determinar los métodos y técnicas de análisis de la información; establecer los criterios y características de los informes que se elaborarán; decidir cómo se difundirán los resultados y quiénes los emplearán para tomar decisiones en relación con el desempeño de los docentes.

Lograr evaluaciones justas y transparentes del desempeño docente para todos los interesados requiere, en gran medida, de procesos inclusivos en los que participen tanto la comunidad académica como los directivos y el personal técnico.

Los objetivos principales de una evaluación de este tipo son aportar evidencias que faciliten y ayuden a la mejora de la enseñanza, contribuir a que los alumnos reciban una mejor educación, y apoyar a las instituciones de educación superior a cumplir los compromisos que tienen con la sociedad de formar profesionales capaces de dar respuestas a las demandas y problemas propios de su campo.

\section{Las funciones de la evaluación del desempeño docente}

Como se mencionó previamente, cuando una institución de educación superior decide evaluar el desempeño de sus profesores, es fundamental que primero se definan claramente los propósitos de este proceso para precisar su naturaleza, es decir, si será de índole diagnóstica, formativa o sumativa. Esta condición perfila la función o funciones que asumirá el proceso evaluativo.

En una evaluación diagnóstica se busca valorar el desempeño de los docentes para fundamentar decisiones de selección de candidatos o para identificar campos de la actuación docente que requieren atención o reforzamientos especiales.

En cambio, una evaluación del desempeño docente con propósito formativo se sustenta en la necesidad de conocer la práctica de los profesores en los escenarios educativos - aulas, talleres, laboratorios, espacios de prácticapara decidir qué aspectos deben reforzarse, mejorarse o modificarse en las dimensiones disciplinarias, pedagógicas, tecnológicas, comunicativas o éticas del ejercicio de los profesores.

Por su parte, las evaluaciones de naturaleza sumativa valoran el desempeño de los docentes en perspectivas globales o integrales, o al término de ciclos definidos con el propósito de apoyar decisiones de naturaleza contractual, como el otorgamiento de la definitividad académica o la promoción en el esquema de nombramientos académicos, entregar premios o reconocimientos o, como ocurre en un gran número de instituciones de educación superior, asignar estímulos compensatorios a los salarios. 


\section{Los modelos para evaluar el desempeño de los docentes}

Para evaluar el desempeño de los docentes es importante identificar el escenario de enseñanza-aprendizaje en el que se encuentran, conocer el contexto en el que están inmersos y determinar los propósitos de la evaluación; de esta forma, se tienen los elementos indispensables para valorar los marcos y procedimientos con los cuales se puede conocer y juzgar el desempeño docente, y que pueden ir desde observaciones sistemáticas hasta el empleo de exámenes sofisticados.

Hoy en día existen diversos modelos para evaluar el desempeño docente (Darling-Hammond, Wise y Pease, 1983; Howard y McColskey, 2001). En este trabajo se describirán los que han sido empleados con mayor frecuencia en las instituciones de educación superior en las últimas décadas, a saber, pares, autoevaluación, opinión de alumnos y portafolios (ver figura 3).

\section{Modelos para evaluar el desempeño docente}

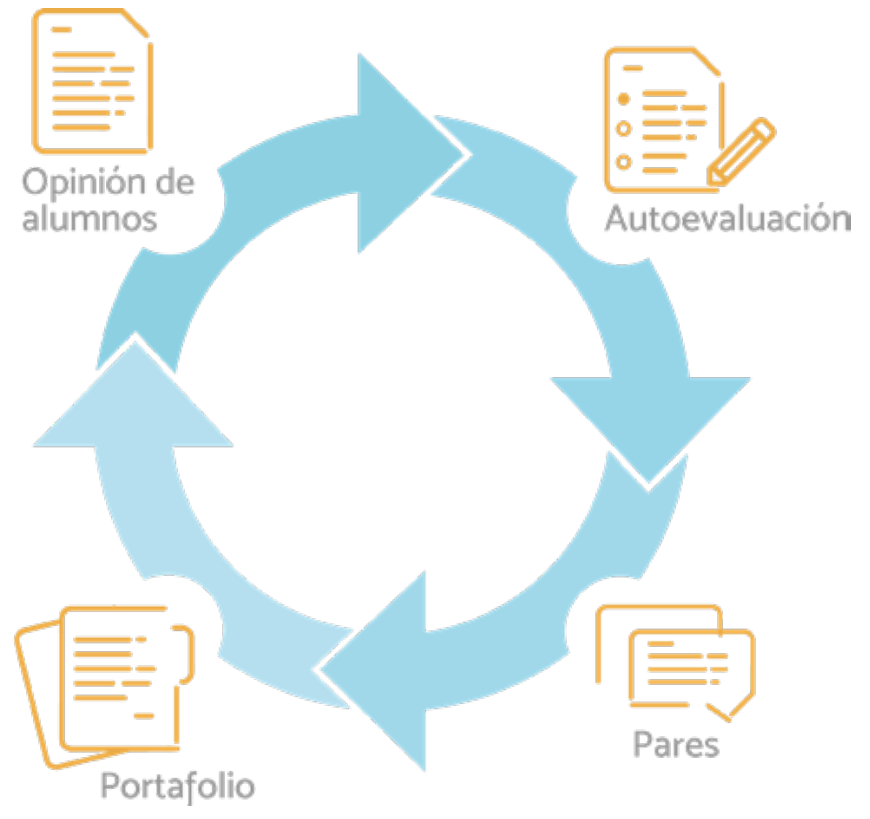

Figura 3. Modelos para evaluar el desempeño docente.

Imagen: Victoria Sánchez. Diseño gráfico: Julián Cruz Cruz.
El modelo de evaluación mediante pares es sin duda el más antiguo de todos, en éste el desempeño es valorado por otros profesores cuya formación y experiencia son similares a la de los docentes evaluados; son expertos en el campo disciplinar y es común que las instituciones integren grupos de tres o cinco pares y que además estén representados académicos de instituciones externas a la de los docentes evaluados. Esta última condición otorga mayor objetividad al proceso, pues una parte de los evaluadores serían totalmente independientes. Una de las principales ventajas es que los evaluadores tienen perfiles académicos similares a los de los evaluados, no obstante, cuando se utiliza este modelo también existe una restricción de los pares externos: que su conocimiento del contexto institucional sería menor, lo que puede derivar en una comprensión restringida del entorno de la evaluación. 
En el caso del modelo de autoevaluación, los docentes evaluados asumen el rol de evaluadores; ellos tienen que juzgar su desempeño apoyándose en guías narrativas, escalas o cuestionarios. En este modelo, resulta decisivo el proceso de reflexión que los docentes tienen que llevar a cabo sobre su formación y actuación en los escenarios educativos, se asume que ellos son quienes mejor pueden juzgar su realidad académica, valorar todos los factores que se enlazan en su desempeño y explorar a profundidad sus procesos y resultados. Este modelo tiene el potencial de estimular el cambio por parte de los docentes; sin embargo, es claro que ser juez y parte en un proceso evaluativo puede restarle credibilidad a éste. Las instituciones que emplean este modelo señalan que es
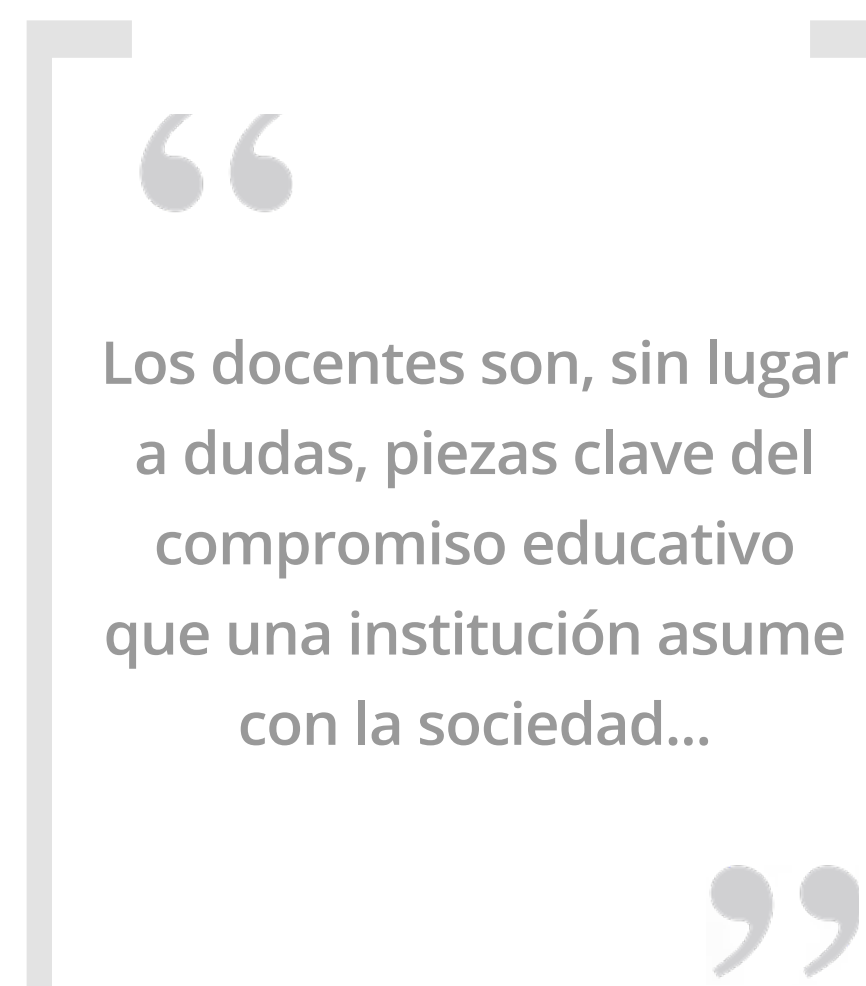

necesario capacitar a los docentes para participar con objetividad en él.

La evaluación del desempeño docente mediante la opinión de los alumnos es, sin duda alguna, el modelo más empleado en la mayoría de las instituciones de educación superior. Su diseño permite obtener la visión y juicio de los beneficiarios de la docencia - los alumnos-, quienes son testigos cotidianos del desempeño de sus profesores. Para evaluar el desempeño de los docentes a través de este modelo, se emplean preponderantemente cuestionarios que integran las acciones que, de acuerdo con la institución, deben realizar los profesores en el escenario de la enseñanza-aprendizaje. En este modelo se obtienen, en poco tiempo, numerosas evidencias de la actuación de los profesores que pueden ser, una vez sistematizadas, contrastadas desde distintos criterios, por ejemplo, docentes de asignaturas teóricas versus prácticas, nivel del ciclo educativo en el que se ubican los docentes, áreas a las que pertenecen, nombramientos, entre otros. Una limitante intrínseca del modelo es que los alumnos sólo pueden valorar lo que ocurre en las aulas, laboratorios, talleres o campos clínicos; no tienen la posibilidad de juzgar otras tareas que se integran a la docencia y que tienen lugar fuera de los escenarios en los que se cristalizan las interacciones entre los docentes y sus alumnos.

Finalmente, la evaluación del desempeño de los docentes a través de portafolios exige un proceso de integración de evidencias y reflexiones que, organizadas a partir de un conjunto de criterios establecido por los líderes del proceso evaluativo, permitirán juzgar el desempeño del profesorado. Este modelo 
"La evaluación del desempeño docente en la educación superior" María Luisa del Carmen Pacheco Cámara, Isela Ibarra Bocardo, Miriam Elizabeth Iñiguez Galindo,

tiene capacidad para combinar aspectos cuantitativos con aspectos cualitativos del desempeño docente, así como para sumar resultados de otras evaluaciones que se hubieran realizado a los docentes; esta perspectiva se presenta como un modelo más integrador y poderoso. Exige que las instituciones determinen muestras válidas de evidencias, periodos de evaluación y grupos de pares que tendrán que intervenir para juzgar el contenido del portafolios que puede manejarse en formatos físicos o virtuales, y exige que los docentes desarrollen una cultura de acopio de testimonios y documentación de experiencias.

Emplear uno o varios modelos para evaluar el desempeño de los profesores dependerá de los propósitos que persigue una institución, del tiempo disponible, así como de los recursos institucionales. Lo que es cierto es que ningún modelo por sí mismo tiene la capacidad para ofrecer una gama completa de valoraciones de las múltiples y variadas tareas que están a cargo del cuerpo académico de las instituciones y que se ponen en juego para cumplir su labor pedagógica.

\section{Los desafíos de la evaluación docente}

Como ya se señaló en este texto, la evaluación del desempeño docente es una práctica que las instituciones de educación superior han abrazado progresivamente. Conforme se ha desarrollado, se han identificado desafíos que afrontan los evaluadores, por lo cual es necesario sensibilizar a quienes desconocen estos procesos; en particular, tener clara la importancia de llevarlos a cabo con profesionalismo para que sean verdaderamente útiles para las instituciones, sus comunidades académicas y para la sociedad.

Es vital que las instituciones reconozcan la evaluación del desempeño docente como una herramienta potencialmente útil, que ofrece sustento a los procesos de toma de decisiones orientados a la mejora de la enseñanza; per se, ninguna evaluación mejora la educación.

Uno de los desafíos centrales de la evaluación radica en que el proceso pueda alcanzar una condición institucional, es decir, que surja por iniciativa de las autoridades y que se legitime ante la comunidad académica por los propósitos que se le asignen y la transparencia con la que se logre plantear su operación.

Lograr prácticas de evaluación del desempeño docente exitosas depende entonces de impulsar procesos precisos, bien fundamentados y guiados o asistidos por especialistas del campo evaluativo.

El diseño y empleo de instrumentos pertinentes y útiles constituye otro desafío en estos procesos, ya que dichas herramientas deben diseñarse a partir de los perfiles de la docencia de las instituciones, para que su contenido responda al entorno institucional. Es esencial que no se empleen instrumentos de otros centros o que sean producto únicamente de una integración de preguntas que no han sido cuidadosamente seleccionadas, juzgadas y probadas. 
Otro desafío tiene que ver con el desarrollo de una verdadera cultura de la evaluación del desempeño de los profesores, que no se limite a obtener y acumular evidencias, sino a transformarlas en decisiones y acciones cuyo impacto se traduzca en el aseguramiento de procesos educativos de calidad.

\section{Las buenas prácticas de evaluación del desempeño de los docentes}

Las Ilamadas buenas prácticas de evaluación del desempeño de los docentes son aquellas que se han instaurado en las instituciones a la luz de acuerdos claros con las comunidades y con propósitos definidos respecto del uso de las evidencias que generarán estos procesos. Son también aquéllas cuyos marcos metodológicos e instrumentales han sido sólidamente desarrollados, examinados y asegurados por profesionales de la evaluación.

Asimismo, se distinguen como buenas prácticas las que de principio a fin y, en forma continua, tienen transparencia y están revestidas de una sólida ética institucional; las prácticas institucionales que se revisan y que se perfeccionan a lo largo del tiempo; y las que transforman las evidencias obtenidas en acciones institucionales claras y efectivas para fortalecer y mejorar el ejercicio profesional de los docentes.

En relación con las buenas prácticas, existen documentos que sistematizan la experiencia de las instituciones con respecto al uso de las evaluaciones, como es el caso de Benton y Young (2018) y, en el mismo sentido, existen marcos más amplios que sirven de apoyo para llevarlas a cabo, como son los Estándares de Evaluación de Personal (The Personnel Evaluation Standards), desarrollado por el Joint Committee on Standards for Educational Evaluation y cuya última revisión data del 2009.

\section{Referencias}

* Benton, S. L. y Young, S. (2018). Best Practices in the Evaluation of Teaching. IDEA Paper, 69, Junio. Recuperado de: http://www.ideaedu.org/Portals/0/Uploads/ Documents/IDEA Papers/IDEA Papers/IDEA Paper 69.pdf.

Coe, R., Aloisi, C., Higgins, S., y Major, L. E. (2014). What makes great teaching? (Review of the underpinning research, Project Report). London: Sutton Trust.

* Darling-Hammond, L., Wise, A. E. y Pease S. R. (1983). Teacher evaluation in the organizational context: a review of the literature. Review of educational research, 53, 285-237. 
* Dewar, K. (2002). On Being a Good Teacher. Journal of Hospitality, Leisure, Sport \& Tourism Education, 1 (1), 61-67.

* Howard, B. B. y McColskey, W. H. (2001). Evaluating Experienced Teachers. Educational Leadership. Evaluating Educators, 58 (5), 48-51.

- The Joint Committee on Standards for Educational Evaluation (2009). The Personnel Evaluation Standards: How to Assess Systems for Evaluating Educators (2 ${ }^{\text {a }}$ edición). (s.l.): SAGE publications.

\section{Cómo citar este artículo}

* Pacheco Cámara, María Luisa del Carmen, Ibarra Bocardo, Isela, Iñiguez Galindo, Miriam Elizabeth, Lee García, Héctor y Victoria Sánchez, Claudia (2018). La evaluación del desempeño docente en la educación superior. Revista Digital Universitaria (RDU). Vol. 19, núm. 6 noviembre-diciembre. DOI: http://doi. org/10.22201/codeic.16076079e.2018.v19n6.a2. 